\title{
Enhanced Expression of miR-34c in Peripheral Plasma Associated with Diabetic Foot Ulcer in Type 2 Diabetes Patients
}

\author{
Tingting $\mathrm{Wu}^{*}$ \\ Dandan Xie* \\ Xiaotong Zhao \\ Murong $\mathrm{Xu}$ (iD \\ Li Luo \\ Datong Deng \\ Mingwei Chen (DD
}

Department of Endocrinology, The First Affiliated Hospital of Anhui Medical University, Hefei, Anhui, 230032, People's Republic of China

*These authors contributed equally to this work
Correspondence: Mingwei Chen Department of Endocrinology, The First Affiliated Hospital of Anhui Medical University, No. 218 Jixi Road, Hefei, Anhui, 230032, People's Republic of China Email chmwl@I63.com
Objective: To explore the correlation between the expression of miR-34c in peripheral blood of patients with type 2 diabetes mellitus (T2DM) and the onset of diabetic foot ulcer (DFU) and diabetic foot osteomyelitis (DFO).

Methods: Sixty newly diagnosed patients with T2DM without DFU (T2DM group), 112 T2DM patients with DFU (DFU group) and 60 controls with normal glucose tolerance (NC group). The DFU group patients were subdivided into DFO $(n=64)$ and NDFO $(n=48)$ groups. Quantitative real-time PCR (qRT-PCR) method was used to determine miR-34c expression levels in the peripheral blood of subjects to analyze the clinical characteristics of DFU and DFO risk factors.

Results: MiR-34c expression level in the T2DM group was marked higher than the NC group [2.99 (1.45-6.22) vs $1.01(0.89-1.52)](\mathrm{P}<0.05)$. However, the expression level of miR-34c in the DFU group was significantly higher than the T2DM group [9.65 (6.15-18.63) vs 2.99 (1.456.22)] $(\mathrm{P}<0.01)$. Compared with the NDFO group, the expression level of miR-34c in the DFO group was also obviously increased $[13.46(8.89-19.11)$ vs $6.02(5.93-14.72)](\mathrm{P}<0.01)$. The expression level of miR-34c in DFU patients was positively correlated with the amputation rate of foot ulcers $(\mathrm{P}=0.030)$ and was negatively correlated with the healing rate of foot ulcers after eight weeks $(\mathrm{P}=0.025)$. Multifactorial logistic regression analysis showed that increased expression of miR-34c was an independent risk factor for $\mathrm{DFU}$ and $\mathrm{DFO}\left(\mathrm{OR}_{\mathrm{DFU}}=3.47, \mathrm{OR}_{\mathrm{DFO}}=4.25\right.$, $\mathrm{P}<0.01)$. Meanwhile, the ROC curve analysis indicated that the AUC of miR-34c for the diagnosis of DFU and DFO was 0.803 and 0.904 , the optimum sensitivity being was $100 \%$ and $98.7 \%$, the optimum specificity was $98.4 \%$ and $98.4 \%$, respectively.

Conclusion: The increased expression of miR-34c in peripheral blood of T2DM patients is closely related to the occurrence, development and prognosis of DFU and DFO.

Keywords: type 2 diabetes, diabetic foot, diabetic foot osteomyelitis, miRNAs

\section{Introduction}

Diabetic foot (DF) is one of the common serious chronic complications of diabetes mellitus (DM). ${ }^{1}$ Diabetic foot ulcer (DFU) is the most common manifestation of DF. Co-infection can make the wound difficult to heal, and then progress to diabetic foot osteomyelitis (DFO), leading to increased mortality, decreased quality of life, and increased risk of lower limb amputation. ${ }^{2}$

MicroRNAs (miRNAs) are a type of endogenous non-coding small RNAs with a length of about 18-25 nucleotides. They regulate gene expression by specifically binding to the $3^{\prime}$ UTR region of downstream target mRNAs. ${ }^{3}$ A body of studies have 
found that the abnormal expression of miRNAs is associated with the occurrence and development of many diseases, including the healing of diabetic foot ulcers and the regulation of bone formation. ${ }^{4,5} \mathrm{MiR}-34 \mathrm{c}$ is a member of the miR-34 family and has been extensively explored in tumor formation, ${ }^{6}$ renal fibrosis, ${ }^{7}$ Alzheimer's disease, ${ }^{8}$ and so forth. Recent studies have shown ${ }^{9}$ that miR-34c can aggravate chronic wound inflammation, inhibit the proliferation and migration of keratinocytes, promote their apoptosis, and hinder the re-epithelialization of wounds. In addition, some studies have also found that miR-34c can participate in the differentiation of osteoblasts and osteoclasts and the process of bone formation. ${ }^{10,11}$ Apart from that, the increased expression of miR-34c in osteoblasts can lead to increased bone loss. ${ }^{12}$ Nowadays, there are few reports about the clinical research on the relationship between miR-34c and the incidence of DFU and DFO. Therefore, we are designed to explain the changes in the miR-34c expression level in the peripheral blood of DFU and DFO patients and their relationship with DFU and the onset and treatment outcome of DFO.

\section{Object and Methods}

\section{Research Subjects}

The subjects were from studies we reported previously. ${ }^{13}$ Briefly, a total of 112 patients with type 2 diabetes (T2DM) and DFU were included from January 2018 to December 2019. The course of the patient's foot ulcer is $\geq 4$ weeks, the ulcer area is within $2-20 \mathrm{~cm}^{2}$ and Wagner's classification is II-IV. Diagnose DFU with osteomyelitis based on medical history, physical signs (especially probe examination), and imaging examination results (X-ray or MRI). Based on this, 112 patients with DFU were labeled into two groups: osteomyelitis group (DFO group, $n=64$ ) and non-osteomyelitis group (NDFO group, $\mathrm{n}=48$ ).

The diabetic group (T2DM group, $n=60$ ) had no DFU, and diabetic lower extremity arteriosclerosis or diabetic peripheral neuropathy. Besides, another 60 healthy individuals who underwent physical examination at the health management center of our hospital were selected as the normal control group (NC group). Subjects in the NC group was received $75 \mathrm{~g}$ oral glucose tolerance test. Normally, a normal test result requires fasting blood glucose (FPG) level is lower than $6.1 \mathrm{mmol} / \mathrm{L}$, and the blood glucose level 2 hours after the glucose load is lower than $7.8 \mathrm{mmol} / \mathrm{L}$. Significantly, exclusion criteria for the patients were with severe heart, liver, and kidney dysfunction, non-cancerous ulcer wounds, autoimmune diseases, and severe sepsis. This study was approved by the Medical Ethics Committee of the First Affiliated Hospital of Anhui Medical University. Meanwhile, this study was obtained from the informed consent of the subjects.

\section{Research Methods}

\section{Treatment of Diabetic Foot Ulcer}

As described by previous study, ${ }^{14}$ all DFU patients were given routine systemic treatment, including anti-infection treatment, antihypertensive treatment, hypoglycemic treatment, correction of hypoproteinemia, neuroprotection treatment, and improvement of blood supply to lower limb wounds, and so on. At the stage of debridement and removing blackened necrotic soft tissue and bone tissue in DFU patients, decompression and negative pressure wound treatment can be given according to the specific situation of DFU patients. At the same time of treatment, the changes in the wound condition of DFU patients should be followed up. For example, after consultation with the diabetic foot multidisciplinary team, it is decided whether to amputation treatment, and the wound healing of DFU patients is recorded after 8 weeks of treatment, etc.

\section{Detection of Observation Indicators}

After all subjects were fasted for 10 hours, the venous blood was drawn from the elbow into the anticoagulant tube (sodium fluoride/EDTA/heparin) or non-anticoagulant tube in the fasted state from 8:00 to 8:30 AM, and different anticoagulants were selected according to the test items. The obtained venous blood samples were used to measure blood glucose, blood lipids, glycosylated hemoglobin (HbA1c), white blood cell (WBC) count, C-reactive protein (CRP), erythrocyte sedimentation rate (ESR), miR$34 \mathrm{c}$, and other indicators. The blood glucose and lipid levels were measured by an automatic biochemical analyzer (MODULE P800, Roche, Switzerland). Fasting blood glucose (FPG) was measured by the glucose oxidase method. Total cholesterol (TCH), triglycerides (TG), highdensity lipoprotein cholesterol (HDL-C), and low-density lipoprotein cholesterol (LDL-C) were detected by oxidaselinked colorimetry. In addition, glycosylated hemoglobin (HbAlc) could be measured by high performance liquid chromatography. C-reactive protein (CRP) was determined by the latex-enhanced scattering immunoturbidimetric 
method. Erythrocyte Sedimentation Rate (ESR) was measured by Weigeldahl method.

The area of wound ulcer was measured using digital photography combined with Image $\mathrm{J}$ analysis software (Image j-ij133-jdk15, USA National Institutes of Health). And then the ankle-brachial index (ABI) was measured by a Doppler blood flow detector (DPL-03, Hangzhou Yuanxiang Medical Equipment Co., Ltd, China). Peripheral vascular disease (PAD) was diagnosed when ABI was less than 0.9 . The percutaneous oxygen partial pressure around ulcers (TcPO2) was determined by a percutaneous oxygen partial pressure detector (TCM 400, Denmark). The measured site should avoid bone protrusion, edema, infection and areas with large blood vessels on the surface, as well as plantar callose. Quantitative real-time PCR (qRT-PCR) was used to determine the expression of miR-34c in peripheral venous blood. More details, $2 \mathrm{~mL}$ of venous blood was collected into an EDTA anticoagulant tube. The obtained plasma was transferred into a $1.5 \mathrm{~mL}$ EP tube without ribonuclease (RNase) and stored at $-80{ }^{\circ} \mathrm{C}$ in a freezer after centrifugation. Finally, the peripheral plasma RNA was extracted following the manufacturer's instructions of miRcute miRNA extraction and isolation kits (Tiangen Biochemical Technology Co., Ltd., Beijing, China).

As we known, the concentration and purity of RNA were determined by ultra-micro ultraviolet spectrophotometry. Moreover, the cDNA was synthesized according to the instructions of using the miRcute miRNA first-strand cDNA synthesis kit (Tiangen Biochemical Technology Co., Ltd., Beijing, China). Further, follow the instructions of the miRcute miRNA fluorescence quantitative detection kit to perform real-time quantitative PCR.

MiR-34c primer sequence: forward, 5'-CGCGTA CATAGTCCTGCCTCTTTGCTGGGGAAA-3'; reverse, 5'- GTACGCGCCCATGTTGCAAAGGGAAATAATCCA3'; U6 primer sequence: forward, 5'-GCTTCGGC AGCACATATACTAAAA-3'; reverse, 5'-CGCTTCACGAA TTTGCCTGTCAT-3'. The set reaction conditions are as follows: a total of 42 cycles comprised pre-denaturation, 95 ${ }^{\circ} \mathrm{C}$ for $2 \mathrm{~min}$; denaturation, $95^{\circ} \mathrm{C}$ for $10 \mathrm{~s}$; annealing, $58^{\circ} \mathrm{C}$ for $20 \mathrm{~s}$; extension, $72^{\circ} \mathrm{C}$ for $10 \mathrm{~s}$. U6 was used as an internal reference. The relative expression of miR-34c was calculated by the $2^{-\Delta \Delta C t}$ method. Each experiment was repeated 3 times independently, and the results were averaged.

\section{Statistical Analysis}

Statistical software SPSS22.0 (Chicago, IL, USA) was used for data analysis. Specifically, normal measurement data is expressed by mean \pm standard deviation, and nonnormal measurement data is expressed by median (interquartile range) $[\mathrm{M}(\mathrm{P} 25, \mathrm{P} 75)]$. In this study, the two groups used the Student's $t$-test, and the comparison between multiple groups used the analysis of variance test. In order to further compare the LSD $t$-test was used. The count data was expressed as a percentage, and the $\chi^{2}$ test was performed. Spearman correlation analysis was used to evaluate the correlation between the expression of $\mathrm{miR}-34 \mathrm{c}$ and other clinical variables. Multivariate unconditional logistic regression was used to understand whether miR-34c is an independent risk factor for DFU and DFO. P values less than 0.05 were considered statistically significant, and all hypothesis tests were two-sided.

\section{Results \\ Comparisons of Clinical Parameters of NC Group, T2DM Group, DFU Group}

The differences in gender composition, age, SBP, DBP, $\mathrm{TCH}$, and LDL-C levels among the NC group, T2DM group and DFU group were not statistically significant $(\mathrm{P}>0.05)$. On the one hand, compared with the NC group, the expression levels of FPG, $\mathrm{HbAlc}, \mathrm{TG}$, and miR-34c in the T2DM group and the DFU group were significantly higher $(\mathrm{P}<0.01)$. Otherwise, the HDL-C level was vitally decreased $(\mathrm{P}<0.01)$. On the other hand, compared with the T2DM group, the duration of diabetes, smoking proportion, FPG, HbA1c, CRP, ESR, WBC count, and miR-34c expression level significantly increased in the DFU group $(\mathrm{P}<0.05)$, while the TcPO2 and $A B I$ levels significantly decreased $(P<0.01)$. From the analysis above, there were no significant differences in SBP, DBP, TG, TCH, LDL-C, and HDL-C levels between the T2DM group and the DFU group $(\mathrm{P}>0.05)$ (Table 1).

\section{Comparisons of Clinical Parameters Between NDFO Group and DFO Group} There were no significant differences between the NDFO group and the DFO group in terms of gender composition, age, DBP, TG, TCH, LDL-C, HDL-C levels, ulcer area, and proportion of PAD $(\mathrm{P}>0.05)$. Compared with the NDFO group, the course of diabetes, foot ulcers duration, Wagner grade, proportion of drug-resistant bacteria infection, SBP, FPG, HbAlc, CRP, ESR levels, WBC count and miR-34c expression levels were dramatically raised in the DFO group. However, TcPO2 and ABI levels were obviously dropped $(\mathrm{P}<0.05)$ (Table 2$)$. 
Table I Comparisons of Clinical Parameters Among NC Group, T2DM Group, and DFU Group [n(\%), ( $\bar{x} \pm s), M(P 25, P 75)]$

\begin{tabular}{|c|c|c|c|c|}
\hline Variable & $N C(n=60)$ & T2DM $(n=60)$ & DFU (n=I|2) & $P$ value \\
\hline Sex & & & & 0.202 \\
\hline Male & $36(60.0)$ & $34(56.7)$ & $60(53.6)$ & \\
\hline Female & $24(40.0)$ & $26(43.3)$ & $52(46.4)$ & \\
\hline Age (year) & $54.7 \pm 10.1$ & $55.1 \pm 11.9$ & $54.5 \pm 10.6$ & 0.473 \\
\hline Smoking (\%) & $16(26.7)$ & $18(30.0)$ & $59(52.7)^{\mathrm{bc}}$ & 0.001 \\
\hline Duration (year) & - & $0.3 \pm 0.2$ & $11.3 \pm 5.2^{\mathrm{d}}$ & $<0.001$ \\
\hline $\mathrm{SBP}(\mathrm{mmHg})$ & $12 \mid \pm 10$ & $126 \pm 14$ & $132 \pm 15$ & 0.087 \\
\hline $\mathrm{DBP}(\mathrm{mmHg})$ & $75 \pm 13$ & $77 \pm 12$ & $82 \pm 14$ & 0.062 \\
\hline FPG $(\mathrm{mmol} / \mathrm{L})$ & $4.8 \pm 0.6$ & $9.9 \pm 2.5^{\mathrm{b}}$ & $11.2 \pm 3.8^{\mathrm{bd}}$ & $<0.001$ \\
\hline $\mathrm{HbAlc}(\%)$ & $5.1 \pm 0.4$ & $8.3 \pm 1.9^{b}$ & $9.1 \pm 2.7^{\mathrm{bc}}$ & $<0.001$ \\
\hline TG (mmol/L) & $1.4 \pm 0.8$ & $1.8 \pm 0.8^{b}$ & $1.7 \pm 0.9^{b}$ & 0.021 \\
\hline $\mathrm{TCH}(\mathrm{mmol} / \mathrm{L})$ & $4.3 \pm 0.8$ & $4.9 \pm 0.7$ & $4.6 \pm 0.7$ & 0.219 \\
\hline LDL-C (mmol/L) & $2.4 \pm 0.3$ & $2.8 \pm 0.5$ & $2.6 \pm 0.5$ & 0.253 \\
\hline HDL-C (mmol/L) & $1.3 \pm 0.2$ & $1.0 \pm 0.3^{\mathrm{a}}$ & $0.9 \pm 0.5^{b}$ & $<0.001$ \\
\hline TcPO2 (mmHg) & $76.4 \pm 7.2$ & $70.5 \pm 8.9$ & $48.5 \pm 10.2^{\text {bd }}$ & $<0.001$ \\
\hline $\mathrm{ABI}$ & $1.12 \pm 0.16$ & $1.05 \pm 0.21$ & $0.84 \pm 0.32^{\mathrm{bc}}$ & $<0.001$ \\
\hline CRP (mg/L) & $7.2 \pm 1.1$ & $9.2 \pm 1.2$ & $48.2 \pm 19.3^{\mathrm{bd}}$ & $<0.001$ \\
\hline WBC $\left(\times 10^{9}\right)$ & $4.2 \pm 0.8$ & $4.7 \pm 0.9$ & $11.2 \pm 4.2^{\mathrm{bd}}$ & $<0.001$ \\
\hline ESR $(\mathrm{mm} / \mathrm{h})$ & $10.2 \pm 2.1$ & $12.7 \pm 3.1$ & $48.2 \pm 19.5^{\mathrm{bd}}$ & $<0.001$ \\
\hline MiR-34c & I.0I (0.89-I.52) & $2.99(1.25-6.22)^{\mathrm{b}}$ & $9.65(4.15-18.63)^{b}$ & $<0.001$ \\
\hline
\end{tabular}

Notes: Data are presented mean \pm standard deviations or numbers (\%) or median with IQR; differences among three groups analyzed using one-way analysis of variance or $x^{2}$ test, and least-significant difference (LSD) analysis was used for comparison between the two groups. Smoking was defined as an average of more than five cigarettes every day for I year. Versus NC group, ${ }^{a} \mathrm{P}<0.05,{ }^{b} \mathrm{P}<0.01$; versus T2DM group, ${ }^{\mathrm{c}} \mathrm{P}<0.05,{ }^{\mathrm{d}} \mathrm{P}<0.01$.

Abbreviations: NC, control group; T2DM, type 2 diabetes group; DFU, diabetic foot ulcer group; Duration, course of diabetes mellitus; SBP, systolic blood pressure; DBP, diastolic blood pressure; FPG, fasting plasma glucose; HbAlc, glycated haemoglobin Alc; TG, triacylglycerol; TCH, total cholesterol; LDL-C, low-density lipoprotein cholesterol; HDL-C, high-density lipoprotein cholesterol; TcPO2, transcutaneous oxygen pressure; ABI, ankle-brachial index; CRP, C-reactive protein; WBC, white blood cell; ESR, erythrocyte sedimentation rate; MiR, microRNA.

\section{Relationship Between miR-34c Expression Level in Peripheral Blood and the Clinical Features of Diabetic Foot Ulcers}

In order to further study the clinical significance of changes in the expression level of miR-34c, we could use the median expression level of miR-34c in the peripheral blood of DFU patients as the dividing point of the group, and furthermore, the low-expression group (lower than the cut-off point) and high-expression group (higher than or equal to the cut-off point). Based on the comparison of the clinical characteristics of the two groups of ulcers, the expression level of miR-34c was negatively correlated with the healing rate of foot ulcers after 8 weeks $(\mathrm{P}=0.025)$ but positively correlated with the course of foot ulcers $(\mathrm{P}=0.027)$. Research data also showed the amputation rate of foot ulcers $(\mathrm{P}=0.030)$. This proves that there is no correlation between the expression level of miR-34c and other clinical features of foot ulcers (Table 3).

\section{Correlations Between Peripheral Blood miR-34c Expression Level and Other Clinical Parameters}

In the T2DM group, the expression of peripheral blood miR-34c was positively correlated with FPG and HbA1c levels $(\mathrm{P}<0.05)$, but there was no significant correlation with other indicators $(\mathrm{P}>0.05)$ as well as $\mathrm{NC}$ group, because no significant correlation was observed between the expression of peripheral blood miR-34c and other clinical parameters either $(\mathrm{P}>0.05)$ (Table 4). On the contrary, in the NDFO group, the expression of peripheral blood miR-34c was positively correlated with ulcer duration, CRP, white blood cell count, and ESR $(\mathrm{P}<0.05)$. But there was no closed correlation with other indicators $(\mathrm{P}>$ 0.05). As for the DFO group, the expression of peripheral blood miR-34c was positively correlated with ulcer duration, Wagner grade, proportion of drug-resistant infection, CRP, WBC counts, and ESR $(\mathrm{P}<0.05)$, but there was no obvious relationship with other indicators $(\mathrm{P}>0.05)$ (Table 5). 


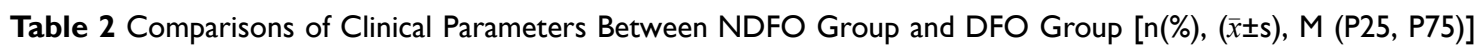

\begin{tabular}{|c|c|c|c|}
\hline & NDFO Group $(n=48)$ & DFO Group $(n=64)$ & $P$ value \\
\hline Sex & & & 0.572 \\
\hline Male & 26 & 34 & \\
\hline Female & 22 & 30 & \\
\hline Age (year) & $53.9 \pm 11.6$ & $54.8 \pm 10.3$ & 0.215 \\
\hline Diabetic duration (year) & $10.8 \pm 5.7$ & $13.9 \pm 6.8^{\mathrm{a}}$ & 0.014 \\
\hline Ulcer duration $(w)$ & $5.1(4.0-10.3)$ & $6.7(4.8-24.2)^{\mathrm{b}}$ & 0.003 \\
\hline Wagner grade & & & 0.039 \\
\hline II & 14 & 0 & \\
\hline III & 28 & 54 & \\
\hline IV & 6 & 10 & \\
\hline Drug-resistant infection (\%) & $20(4 \mid .7)$ & $39(60.9)$ & 0.043 \\
\hline $\mathrm{SBP}(\mathrm{mm} \mathrm{Hg})$ & $128 \pm 12$ & $138 \pm 16^{a}$ & 0.018 \\
\hline $\mathrm{DBP}(\mathrm{mm} \mathrm{Hg})$ & $83 \pm 14$ & $87 \pm 15$ & 0.072 \\
\hline FPG (mmol/L) & $10.3 \pm 2.8$ & $12.6 \pm 3.6^{\mathrm{a}}$ & 0.035 \\
\hline HbAlc (\%) & $8.8 \pm 2.2$ & $9.3 \pm 3.1^{\mathrm{a}}$ & 0.028 \\
\hline TG (mmol/L) & $1.7 \pm 0.8$ & $1.8 \pm 0.9$ & 0.503 \\
\hline $\mathrm{TCH}(\mathrm{mmol} / \mathrm{L})$ & $4.7 \pm 0.8$ & $4.5 \pm 0.7$ & 0.492 \\
\hline LDL-C (mmol/L) & $2.8 \pm 0.7$ & $2.6 \pm 0.8$ & 0.488 \\
\hline HDL-C (mmol/L) & $1.0 \pm 0.2$ & $0.9 \pm 0.3$ & 0.571 \\
\hline TcPO2 (mm Hg) & $56.9 \pm 8.9$ & $38.4 \pm 10.5^{\mathrm{a}}$ & 0.016 \\
\hline $\mathrm{ABI}$ & $0.86 \pm 0.31$ & $0.77 \pm 0.24^{\mathrm{a}}$ & 0.031 \\
\hline PAD (\%) & $26(54.2)$ & $40(62.5)$ & 0.375 \\
\hline CRP (mg/dl) & $32.2 \pm 15.7$ & $63.2 \pm 20.6^{\mathrm{b}}$ & $<0.001$ \\
\hline WBC $\left(\times 10^{9}\right)$ & $10.1 \pm 3.7$ & $13.4 \pm 4.8 \mathrm{~b}$ & 0.002 \\
\hline ESR $(\mathrm{mm} / \mathrm{h})$ & $38.9 \pm 16.4$ & $70.8 \pm 21.1^{b}$ & $<0.001$ \\
\hline Ulcer area $\left(\mathrm{cm}^{2}\right)$ & $10.3 \pm 3.4$ & $9.3 \pm 3.6$ & 0.276 \\
\hline MiR-34c & $6.02(5.93-14.72)$ & $13.46(8.89-19.11)^{b}$ & $<0.001$ \\
\hline
\end{tabular}

Notes: Data are presented mean \pm standard deviations or numbers (\%) or median with IQR; differences between two groups analyzed using $t$-test or $x^{2}$ test. The data of drug-resistant infection were derived from the microbial drug sensitivity results of DFO group and NDFO group versus the NDFO group, ${ }^{\text {a }}<<0.05$, ${ }^{b} P<0.01$.

Abbreviations: NDFO, diabetic foot ulcer without osteomyelitis; DFO, diabetic foot osteomyelitis; SBP, systolic blood pressure; DBP, diastolic blood pressure; FPG, fasting plasma glucose; HbAIc, glycated hemoglobin AIc; TG, triacylglycerol; TCH, total cholesterol; LDL-C, low-density lipoprotein cholesterol; HDL-C, high-density lipoprotein cholesterol; TcPO2, transcutaneous oxygen pressure; ABI, ankle brachial index; PAD, peripheral vascular disease; CRP, C-reactive protein; WBC, white blood cell; ESR, erythrocyte sedimentation rate; MiR, MicroRNA.

\section{Analysis of Risk Factors for a Diabetic Foot Ulcer and Diabetic Foot Osteomyelitis}

In diabetic patients, multifactorial unconditional logistic regression analysis was performed with DFU as the dependent variable and gender, age, smoking, diabetic duration, ulcer duration, Wagner grade, SBP, DBP, FPG, HbA1c, TG, TCH, LDL-C, HDL-C, TcPO2, ABI, CRP, $\mathrm{WBC}$, ESR, ulcer area, and miR-34c as independent variables, respectively. One interesting point has to note that the duration of diabetes, HbAlc, TcPO2, $\mathrm{CRP}$, and high expression of miR-34c were all independent risk factors for DFU (Table 6). Also, in DFU patients, multifactorial unconditional logistic regression analysis was performed with DFO as the dependent variable and gender, age, diabetic duration, ulcer duration, Wagner grade, drug-resistant infection, SBP, DBP, FPG, HbA1c, TG, TCH, LDL-C, HDL-C, TcPO2, ABI, $\mathrm{CRP}, \mathrm{WBC}$, ESR, ulcer area, and miR-34c as independent variables. The final analysis indicated that independent risk factors for DFO included ulcer course duration, HbAlc, ABI, WBC count, ESR, and high expression of miR-34c (Table 7).

\section{Mark Verification}

To further explore the potential value of miR-34c in the diagnosis of DFU, this trial takes the following steps: We will purposefully select 158 subjects (46 cases of T2DM and 
Table 3 Relationship Between the Expression Levels of miR-34c in Peripheral Blood and the Clinical Features of DFU Patients [( $\bar{x} \pm \mathrm{s}), \mathrm{n}(\%)]$

\begin{tabular}{|c|c|c|c|}
\hline & $\begin{array}{l}\text { High Expression } \\
\text { Group }(n=46)\end{array}$ & $\begin{array}{l}\text { Low Expression } \\
\text { Group }(n=66)\end{array}$ & $P$ value \\
\hline Age (y) & $54.4 \pm 11.7$ & $55.2 \pm 10.4$ & 0.302 \\
\hline $\begin{array}{l}\text { Sex } \\
\qquad \text { Male } \\
\text { Female }\end{array}$ & $\begin{array}{l}24(52.2) \\
22(47.8)\end{array}$ & $\begin{array}{l}36(54.5) \\
30(45.5)\end{array}$ & 0.804 \\
\hline $\begin{array}{l}\text { Ulcer area }\left(\mathrm{cm}^{2}\right) \\
\leq 5 \\
5 \sim 10 \\
>10\end{array}$ & $\begin{array}{l}5(10.9) \\
26(56.5) \\
15(32.6)\end{array}$ & $\begin{array}{l}12(18.2) \\
43(65.1) \\
11(16.7)\end{array}$ & 0.119 \\
\hline $\begin{array}{l}\text { Ulcer duration }(w) \\
\leq 6 \\
6 \sim 10 \\
>10\end{array}$ & $\begin{array}{l}8(17.4) \\
23(50.0) \\
15(32.6)\end{array}$ & $\begin{array}{l}18(27.3) \\
40(60.6) \\
8(12.1)\end{array}$ & 0.027 \\
\hline $\begin{array}{l}\text { Wagner grade } \\
\text { II } \\
\text { III } \\
\text { IV }\end{array}$ & $\begin{array}{l}5(10.9) \\
31(67.4) \\
10(21.7)\end{array}$ & $\begin{array}{l}9(13.6) \\
51(77.3) \\
6(9.1)\end{array}$ & 0.168 \\
\hline $\begin{array}{l}\text { Ulcer healing rate } \\
\text { after } 8 \text { weeks (\%) } \\
\text { Healing } \\
\text { Non-healing }\end{array}$ & $\begin{array}{l}18(39.1) \\
28(60.9)\end{array}$ & $\begin{array}{l}40(60.6) \\
26(39.4)\end{array}$ & 0.025 \\
\hline $\begin{array}{l}\text { Amputation rate (\%) } \\
\text { Amputation } \\
\text { Non-amputation }\end{array}$ & $\begin{array}{l}10(21.7) \\
36(78.3)\end{array}$ & $\begin{array}{l}5(7.6) \\
61(92.4)\end{array}$ & 0.030 \\
\hline
\end{tabular}

Notes: Data are presented mean \pm standard deviations or numbers (\%); differences between two groups analyzed using $t$-test or $x^{2}$ test. The cut-off point of miR-34c expression level for grouping was 9.65 .

112 cases of DFU), and doctors will measure them during the experiment. The patient's peripheral blood miR-34c was expressed, and the ROC curve was determined to evaluate the sensitivity and specificity of miR-34c in diagnosing DFU. The results showed that the AUC of miR-34c for diagnosing DFU was 0.803 (95\% CI, 0.618-0.879, $\mathrm{P}<0.001)$, the best cut-off point of miR-34c was 2.11 , the sensitivity was $100 \%$, and the specificity was $98.4 \%$ (Figure 1A). Similarly, to explore the potential value of miR-34c in the diagnosis of DFO, we also selected 112 subjects (48 cases of non-DFO and 64 cases of DFO) with the purpose, and used ROC curve to evaluate the sensitivity and specificity of miR-34c expression level in diagnosing DFO. Based on experimental investigation, we found that the AUC of miR-34c in the diagnosis of DFO was 0.904 ( $95 \%$ CI, $0.852-0.955, \mathrm{P}<0.001)$, and the obtained sensitivity and specificity were $98.7 \%$ and $98.4 \%$
Table 4 Correlations Between miR-34c Expression Levels and Other Clinical Parameters in NC Group and T2DM Group ( $r$ )

\begin{tabular}{|l|l|l|l|l|}
\hline \multirow{2}{*}{ Variables } & \multicolumn{2}{|c|}{ T2DM (n =60) } & \multicolumn{2}{c|}{ NC (n =60) } \\
\cline { 2 - 5 } & $\mathbf{r}$ & P value & $\mathbf{r}$ & P value \\
\hline Age & 0.003 & 0.983 & 0.036 & 0.482 \\
Sex & -0.017 & 0.843 & 0.112 & 0.146 \\
Diabetic duration & 0.069 & 0.502 & - & - \\
Smoking & 0.086 & 0.473 & 0.063 & 0.539 \\
SBP & 0.102 & 0.216 & 0.103 & 0.169 \\
DBP & 0.091 & 0.791 & 0.018 & 0.683 \\
FPG & 0.198 & 0.041 & 0.078 & 0.341 \\
HbAlc & 0.187 & 0.048 & 0.066 & 0.435 \\
TG & 0.091 & 0.295 & -0.011 & 0.788 \\
TCH & -0.020 & 0.822 & -0.026 & 0.647 \\
LDL-C & 0.005 & 0.957 & 0.052 & 0.522 \\
HDL-C & -0.046 & 0.652 & -0.072 & 0.353 \\
TCPO2 & -0.085 & 0.397 & -0.009 & 0.652 \\
ABI & -0.105 & 0.198 & 0.156 & 0.104 \\
CRP & 0.023 & 0.802 & 0.082 & 0.328 \\
WBC & 0.078 & 0.421 & -0.008 & 0.827 \\
ESR & 0.102 & 0.203 & -0.098 & 0.275 \\
\hline
\end{tabular}

Abbreviations: NC, control group; T2DM, type 2 diabetes group; SBP, systolic blood pressure; DBP, diastolic blood pressure; FPG, fasting plasma glucose; HbAlc, glycated hemoglobin A Ic; TG, triacylglycerol; TCH, total cholesterol; LDL-C, lowdensity lipoprotein cholesterol; HDL-C, high-density lipoprotein cholesterol; TcPO2, transcutaneous oxygen pressure; $A B I$, ankle brachial index; CRP, C-reactive protein; WBC, white blood cell; ESR, erythrocyte sedimentation rate; MiR, MicroRNA.

with the optimal cut-off point of miR-34c being 5.95 (Figure 1B).

\section{Discussion}

Based on the above research, it is easy to find that the miR-34c expression level in peripheral blood of newly diagnosed T2DM patients is significantly higher than that of people with normal glucose tolerance. In addition, miR$34 \mathrm{c}$ level in DFU patients was also significantly higher than that of patients without DFU. But it has to be said on condition that the foot ulcer infection progresses to diabetic foot osteomyelitis, the expression level of miR-34c will sharp increase as well. Therefore, the reason is that the high miR-34c expression is an independent risk factor for DFU and DFO. This can easily enhance the expression level of miR-34c in peripheral blood of T2DM patients and the healing rate of foot ulcers and the rate of amputation. DFU with high expression of miR-34c has a lower healing rate and a higher risk of amputation, indicating that the high expression of miR-34c is not only a strong 
Table 5 Correlations Between miR-34c Expression Levels and Other Clinical Parameters in the NDFO Group and DFO Group

\begin{tabular}{|l|l|l|l|l|}
\hline \multirow{2}{*}{ Variables } & \multicolumn{2}{|c|}{ NDFO (n =48) } & \multicolumn{2}{c|}{ DFO (n =64) } \\
\cline { 2 - 5 } & $\mathbf{r}$ & $\boldsymbol{P}$ value & $\mathbf{r}$ & $\boldsymbol{P}$ value \\
\hline Age & 0.018 & 0.783 & 0.064 & 0.574 \\
Sex & 0.043 & 0.617 & 0.102 & 0.328 \\
Diabetic duration & 0.163 & 0.102 & 0.178 & 0.085 \\
Ulcer duration & 0.193 & 0.041 & 0.193 & 0.041 \\
Wagner grade & 0.111 & 0.182 & 0.335 & 0.004 \\
Drug-resistant infection & 0.174 & 0.084 & 0.265 & 0.017 \\
SBP & 0.183 & 0.072 & 0.084 & 0.298 \\
DBP & 0.084 & 0.524 & 0.076 & 0.603 \\
FPG & 0.166 & 0.098 & 0.171 & 0.082 \\
HbAIc & 0.187 & 0.063 & 0.165 & 0.107 \\
TG & 0.014 & 0.826 & 0.068 & 0.546 \\
TCH & 0.068 & 0.719 & -0.082 & 0.658 \\
LDL-C & 0.096 & 0.428 & 0.122 & 0.239 \\
HDL-C & -0.134 & 0.158 & -0.167 & 0.141 \\
TcPO2 & -0.098 & 0.787 & -0.173 & 0.087 \\
ABI & -0.117 & 0.179 & -0.155 & 0.114 \\
CRP & 0.197 & 0.039 & 0.302 & 0.005 \\
WBC & 0.264 & 0.019 & 0.297 & 0.011 \\
ESR & 0.391 & $<0.001$ & 0.352 & 0.002 \\
Ulcer area & 0.183 & 0.059 & 0.191 & 0.055 \\
\hline NG: & & & \\
\hline
\end{tabular}

Notes: The data of drug-resistant infection were derived from the microbial drug sensitivity results of DFO group and NDFO group.

Abbreviations: NDFO, diabetic foot ulcer without osteomyelitis; DFO, diabetic foot osteomyelitis; SBP, systolic blood pressure; DBP, diastolic blood pressure; FPG, fasting plasma glucose; HbAlc, glycated hemoglobin Alc; TG, triacylglycerol; $\mathrm{TCH}$, total cholesterol; LDL-C, low-density lipoprotein cholesterol; HDL-C, high-density lipoprotein cholesterol; TcPO2, transcutaneous oxygen pressure; $A B I$, ankle brachial index; CRP, C-reactive protein; WBC, white blood cell; ESR, erythrocyte sedimentation rate; MiR, MicroRNA.

risk factor for the onset of DFU and DFO but also as a potential biomarker for the prognosis of DFU and DFO treatment. To our knowledge, this is the first study to investigate the relationship between changes in miR-34c expression and the pathogenesis and treatment outcomes of DFU and DFO in patients with T2DM.

In the present study, peripheral blood miR-34c levels were notably enhanced in patients with T2DM compared with control individuals with normal glucose tolerance. Correlation analysis showed that in T2DM patients, miR$34 \mathrm{c}$ expression was correlated with FPG and HbA1c positively. ${ }^{15}$ Animal studies have also found that high miR-34c expression facilitated visceral fat accumulation in cU2 transgen, caused insulin resistance, and impaired glucose tolerance. Though miR-34a and miR-34c are homologues of the miR-34 family. Kong et al ${ }^{16}$ discovered that peripheral serum miR-34a levels were more remarkably improved than those of prediabetes and normal
Table 6 The Multiple Logistic Regression Analysis of Risk Factors for Diabetic Foot Ulcers

\begin{tabular}{|l|l|l|l|l|l|l|}
\hline Variable & $\boldsymbol{\beta}$ & SE & Wald & OR & $\mathbf{9 5 \% ~ C I}$ & $\boldsymbol{P}$ value \\
\hline $\begin{array}{l}\text { Diabetic } \\
\text { duration (y) }\end{array}$ & 0.67 & 0.37 & 11.12 & 5.12 & $1.19 \sim 13.76$ & $<0.001$ \\
\hline HbAIc (\%) & 0.43 & 0.41 & 3.04 & 1.29 & $1.03 \sim 9.12$ & 0.031 \\
\hline $\begin{array}{l}\text { TcPO2 } \\
\text { (mmHg) }\end{array}$ & 0.55 & 0.35 & 4.78 & 1.76 & $1.18 \sim 10.13$ & 0.013 \\
\hline CRP (mg/L) & 0.36 & 0.33 & 2.82 & 1.13 & $1.03 \sim 9.71$ & 0.043 \\
\hline miR-34c & 0.74 & 0.41 & 7.43 & 3.47 & $1.18 \sim 12.96$ & 0.001 \\
\hline
\end{tabular}

Notes: Multivariate unconditional logistic regression analysis adjusted for sex, age, smoking, diabetic duration, ulcer duration, Wagner grade, SBP, DBP, FPG, HbAIc, TG, TCH, LDL-C, HDL-C, TCPO2, ABI, CRP, WBC count, ESR, ulcer area, miR34c.

Abbreviations: SBP, systolic blood pressure; DBP, diastolic blood pressure; FPG, fasting plasma glucose; HbAlc, glycated hemoglobin Alc; TG, triacylglycerol; $\mathrm{TCH}$, total cholesterol; LDL-C, low-density lipoprotein cholesterol; HDL-C, high-density lipoprotein cholesterol; TcPO2, transcutaneous oxygen pressure; $\mathrm{ABI}$, ankle brachial index; CRP, C-reactive protein; WBC, white blood cell; ESR, erythrocyte sedimentation rate; MiR, MicroRNA.

Table 7 The Multiple Logistic Regression Analysis of Risk Factors for Diabetic Foot Osteomyelitis

\begin{tabular}{|l|l|l|l|l|l|l|}
\hline Variable & $\boldsymbol{\beta}$ & SE & Wald & OR & 95\% CI & P value \\
\hline Ulcer duration (w) & 0.85 & 0.38 & 9.12 & 3.24 & $1.13 \sim 10.45$ & 0.001 \\
\hline HbAIc (\%) & 0.62 & 0.35 & 6.26 & 2.09 & $1.18 \sim 8.35$ & 0.016 \\
\hline $\mathrm{ABI}$ & 0.43 & 0.26 & 3.29 & 1.29 & $1.03 \sim 9.12$ & 0.032 \\
\hline $\begin{array}{l}\text { WBC count } \\
\left(\times 10^{9}\right)\end{array}$ & 0.37 & 0.34 & 3.15 & 1.21 & $1.22 \sim 11.98$ & 0.042 \\
\hline ESR (mm/h) & 0.51 & 0.32 & 4.02 & 2.01 & $1.11 \sim 10.78$ & 0.007 \\
\hline miR-34c & 0.76 & 0.26 & 6.28 & 4.25 & $1.16 \sim 13.22$ & $<0.001$ \\
\hline
\end{tabular}

Notes: Multivariate unconditional logistic regression analysis adjusted for sex, age, diabetic duration, ulcer duration, Wagner grade, SBP, DBP, FPG, HbAlc, TG, TCH, LDL-C, HDL-C, TcPO2, ABI, CRP, WBC, ESR, ulcer area, and miR-34c.

Abbreviations: SBP, systolic blood pressure; DBP, diastolic blood pressure; FPG, fasting plasma glucose; HbAlc, glycated hemoglobin Alc; TG, triacylglycerol; TCH, total cholesterol; LDL-C, low-density lipoprotein cholesterol; HDL-C, high-density lipoprotein cholesterol; TcPO2, transcutaneous oxygen pressure; $\mathrm{ABI}$, ankle brachial index; CRP, C-reactive protein; WBC, white blood cell; ESR, erythrocyte sedimentation rate; MiR, MicroRNA.

glucose tolerance people in newly diagnosed T2DM patients. Therefore, these findings support our research hypothesises. However, some studies have also found that the expression of miR-34c in peripheral blood mononuclear cells of T2DM patients is sharply reduced, and low expression of miR-34c is a non-negligible risk factor for T2DM. ${ }^{17}$ This result is inconsistent with the results of this 
A

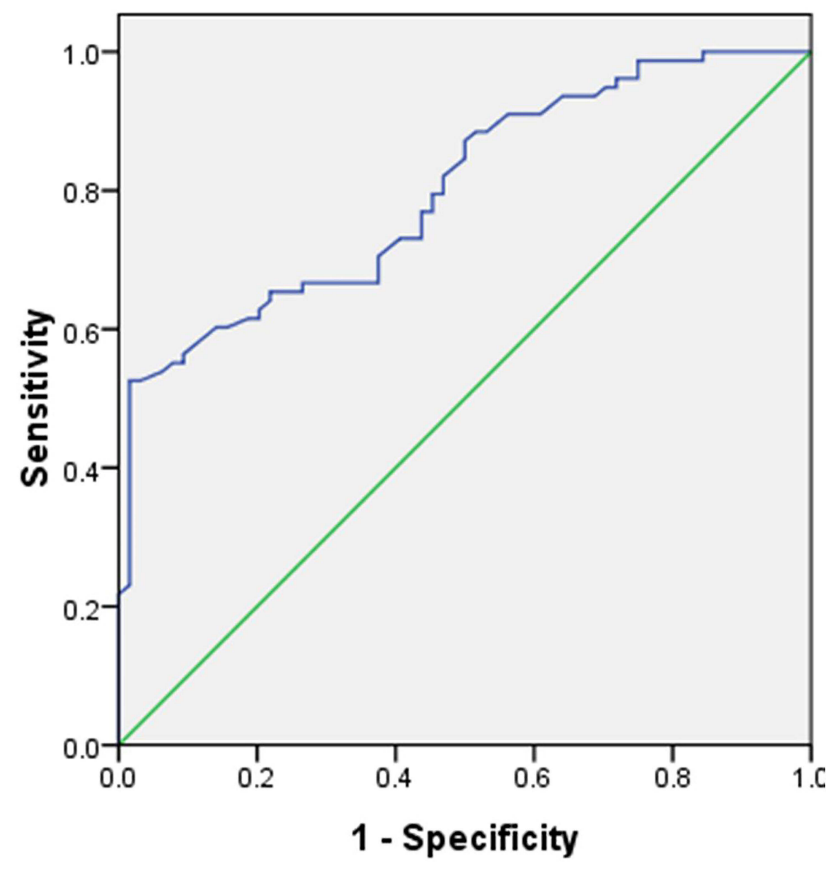

B

ROC Curve

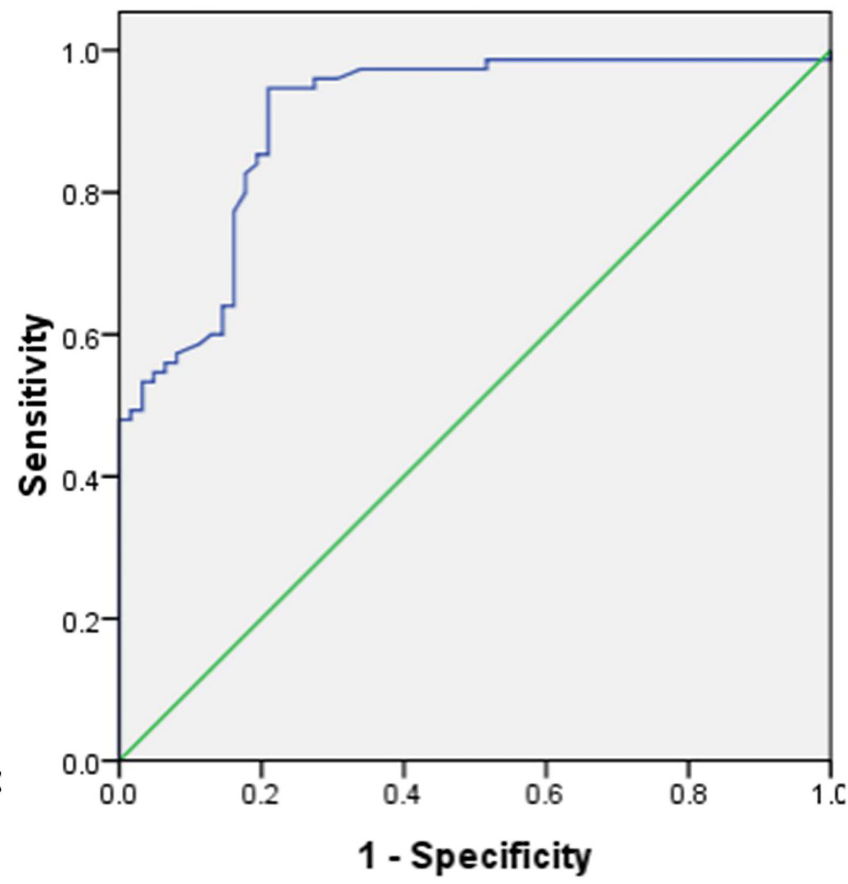

Figure I The biomarker potential of circulating miR-34c for DFU, DFO, and control. ROC analysis was used to evaluate the ability of circulating miR-34c to distinguish between different groups. (A) MiR-34c distinguished DFU patients from controls with area under curve (AUC) of 0.803 (95\% Cl, 0.6I8-0.879, P<0.00I). (B) MiR-34c distinguished DFO patients from DFU patients with AUC of $0.904(95 \% \mathrm{Cl}, 0.852-0.955, \mathrm{P}<0.00 \mathrm{I})$.

Abbreviations: DFU, diabetic foot ulcer; DFO, diabetic foot osteomyelitis.

study. We speculate that the reason is different from the selected research object and the source of measured miR$34 \mathrm{c}$ expression. Many researches have also been demonstrated that wound inflammation is a key factor affecting circulation miRNA expression. ${ }^{18}$ Several studies supporting our findings revealed that miR-34c expression can be significantly upregulated in patients with a pathogen infection state. ${ }^{19,20}$

In this study, the duration of the foot ulcer in the DFU group was at least 4 weeks, which was a chronic, difficult-to -heal wound. The clinical features include a long history of diabetes, poor long-term blood sugar control, high proportion of smoking, combined with varying degrees of abnormal lipid metabolism, peripheral vascular disease, and infectious inflammation. Multivariate regression analysis showed that the course of diabetes, $\mathrm{HbA} 1 \mathrm{c}, \mathrm{TcPO} 2$, and $\mathrm{CRP}$ were independent factors influencing the occurrence of foot ulcers, which were consistent with previous research results. ${ }^{21-23}$ However, in this study, we did not find that smoking is an independent factor affecting the incidence of DFU, which is inconsistent with previous studies. ${ }^{24}$ It is speculated that other factors in this study may have a higher impact on the development of DFU than smoking. Further analysis found that compared with the T2DM group, the peripheral blood miR-34c level of the DFU group was observably improved; the miR-34c expression level was positively correlated with the course of ulcer, and negatively correlated with the ulcer healing rate after 8 weeks. Multivariate regression analysis showed that highly expressed miR-34c is an independent risk factor for foot ulcers. It is suggested that miR-34c may be involved in the occurrence of diabetic foot ulcers and can be used as a marker for the prognosis of DFU. At present, it is generally believed that the persistent and excessive inflammatory state in the wound and the impaired function of keratinocytes in the epidermis are important influencing factors that make DFU difficult to heal. ${ }^{25} \mathrm{Wu}$ et $\mathrm{al}^{9}$ reported that the expression of miR-34c increases significantly in keratinocytes at the margins of venous ulcer wounds. For one thing, it induces the secretion of a large number of proinflammatory cytokines and chemical factors, resulting in excessive neutrophils and giant cells. The continuous accumulation of phages will aggravate the inflammation of chronic wounds, which is not conducive to the elimination of infection. For another, the up-regulation of miR-34c 
expression can also inhibit the proliferation and migration of keratinocytes and promote their apoptosis.

Further analysis found that compared with simple foot ulcers, patients with foot ulcers combined with osteomyelitis had a longer duration of diabetes and foot ulcers, a higher Wagner grade of foot ulcers, poorer glycaemic control status, as well as worse limb blood supply status, and more severe infection along with higher proportion of drug-resistant infection. Multivariate regression analysis showed that the courses of foot ulcer, HbA1c, ABI, WBC count, and ESR are all independent factors influencing the occurrence of foot ulcer with osteomyelitis, which is consistent with previous research results. ${ }^{26-28}$ It should be noted that in this study, we did not find that drugresistant infection is an independent factor affecting the onset of DFO, which is inconsistent with previous studies. ${ }^{29}$ Presumably, other factors in this study may be more influential than the drug-resistant infection on the development of DFO. More details, further analysis illustrated that the peripheral blood miR-34c level in the DFO group was further increased compared with patients in the NDFO group; the expression level of miR-34c was extremely correlated with the amputation rate of foot ulcers. Multivariate regression analysis showed that high expression of miR-34c is an independent risk factor for foot ulcers with osteomyelitis. Meanwhile, it suggestted that miR-34c might be involved in the occurrence of DFO and can be used as a marker for the risk of DFO amputation. DFO is mostly chronic osteomyelitis, bone destruction and dead bone formation due to infection. Therefore, the differentiation characteristics of osteoblasts and their osteogenic capacity are crucial for the repair of bone injury and can directly affect the clinical outcome of osteomyelitis. $^{30}$ Numerous studies have confirmed that miR-34c plays a vital role in the regulation of bone metabolism. ${ }^{11,31,32}$ In animal studies, ${ }^{33}$ increased miR$34 \mathrm{c}$ expression in osteoblasts resulted in reduced osteogenesis capacity and age-related osteoporosis in mice. In vitro experiments ${ }^{12}$ found that lipopolysaccharide (LPS) could significantly upregulate the expression of miR-34c in osteoblasts and promote bone resorption. In contrast, Icariin could restore LPS-induced bone loss by downregulating miR-34c expression levels.

It turned out that the expression level of miR-34c in peripheral blood could be used as a biomarker for the prediction and diagnosis of Alzheimer's disease ${ }^{8,34}$ as well as for vulnerability to T2DM and insulin resistance secondary to stress reaction. ${ }^{17}$ Beyond that, according to the results of ROC curve analysis, we also pointed that the expression level of miR-34c in peripheral blood of T2DM patients could serve as a potential biomarker for the prediction of DFU and DFO. What is more, we also discovered that the expression level of miR-34c was positive correlation with the course of DFU and amputation rate. On the contrary, it also represents negative correlation with the healing rate of DFU after eight weeks. Hence, the forementioned results illustrate the functionality of expression level of miR-34c in peripheral blood for the diagnosis and prognosis of DFU and DFO. Nonetheless, further studies are needed to identify the reasons for the increased expression of miR-34c in peripheral blood of patients with DFU and DFO.

In conclusion, this study found that the increased level of miR-34c expression in peripheral blood of type 2 diabetes patients was closely related to the occurrence, development and prognosis of DFU. Yet when it comes to the shortcomings of this study, the main shortcoming as follows: a single-center study with a small sample size, and selection bias. Secondly, this study was unable to clarify the causal relationship between miR-34c and the development of DFU and DFO. Notably, it was reported that genetic polymorphisms are related to the risk of DF. ${ }^{35}$ There need more studies to further confirm the cause of increased miR-34c in peripheral blood in patients with DFU and DFO in the future to explore the mechanism of miR-34c and to assess whether the genetic susceptibility between miR-34c and the development of DFU and DFO, and miR-34c could act as a new therapeutic target for the treatment of DFO.

\section{Abbreviations}

DFO, diabetic foot osteomyelitis; NDFO, diabetic foot ulcer without osteomyelitis; AUC, area under the curves; $\mathrm{ABI}$, ankle brachial index; FPG, fasting plasma glucose; HbA1c, glycated hemoglobin A1c; WBC, white blood cell; CRP, C-reactive protein; ESR, erythrocyte sedimentation rate; $\mathrm{TCH}$, total cholesterol; $\mathrm{TG}$, triacylglycerol; HDL-C, high-density lipoprotein cholesterol; LDL-C, lowdensity lipoprotein cholesterol; TcPO2, transcutaneous oxygen pressure; SBP, systolic blood pressure; DBP, diastolic blood pressure.

\section{Data Sharing Statement}

The datasets used and/or analyzed during the current study are available from the corresponding author upon reasonable request. 


\section{Ethical Approval}

All procedures were performed in studies involving human participants in accordance with the ethical standards of the institutional and/or national research committee and with the 1964 Helsinki declaration and its later amendments or comparable ethical standards.

\section{Acknowledgments}

We are grateful to the all patients for participating in the study. We thank the participants of this study including medical personnel, and researchers from the Department of Endocrinology in the First Affiliated Hospital of Anhui Medical University.

\section{Author Contributions}

All authors made a significant contribution to the work reported, whether that is in the conception, study design, execution, acquisition of data, analysis and interpretation, or in all these areas; took part in drafting, revising or critically reviewing the article; gave final approval of the version to be published; have agreed on the journal to which the article has been submitted; and agree to be accountable for all aspects of the work.

\section{Funding}

This study was supported by grants from the Key Research and Development Program of Anhui Province (grant no. 202004a07020016) and the Natural Science Foundation of Anhui Province in China (2108085MH269).

\section{Disclosure}

The authors report no conflicts of interest in this work.

\section{References}

1. Woodbury MG. Diabetic foot risk assessment. Diabetes Metab Res Rev. 2016;32(4):376-378. doi:10.1002/dmrr.2784

2. Pitocco D, Spanu T, Di Leo M, et al. Diabetic foot infections: a comprehensive overview. Eur Rev Med Pharmacol Sci. 2019;23(2 Suppl):26-37.

3. Krol J, Loedige I, Filipowicz W. The widespread regulation of microRNA biogenesis, function and decay. Nat Rev Genet. 2010;11 (9):597-610. doi:10.1038/nrg2843

4. Goodarzi G, Maniati M, Qujeq D. The role of microRNAs in the healing of diabetic ulcers. Int Wound J. 2019;16(3):621-633. doi:10.1111/iwj.13070

5. van Wijnen AJ, van de Peppel J, van Leeuwen JP, et al. MicroRNA functions in osteogenesis and dysfunctions in osteoporosis. Curr Osteoporos Rep. 2013;11(2):72-82. doi:10.1007/s11914-013-0143-6

6. Maroof H, Salajegheh A, Smith RA, Lam AK. MicroRNA-34 family, mechanisms of action in cancer: a review. Curr Cancer Drug Targets. 2014;14(8):737-751. doi:10.2174/1568009614666141020100337
7. Morizane R, Fujii S, Monkawa T, et al. miR-34c attenuates epithelial-mesenchymal transition and kidney fibrosis with ureteral obstruction. Sci Rep. 2014;4(1):4578. doi:10.1038/srep04578

8. Bhatnagar S, Chertkow H, Schipper HM, et al. Increased microRNA$34 \mathrm{c}$ abundance in Alzheimer's disease circulating blood plasma. Front Mol Neurosci. 2014;7:2. doi:10.3389/fnmol.2014.00002

9. Wu J, Li X, Li D, et al. MicroRNA-34 family enhances wound inflammation by targeting LGR4. J Invest Dermatol. 2020;140 (2):465-476.e11. doi:10.1016/j.jid.2019.07.694

10. Liu Y, Xu F, Pei HX, et al. Vaspin regulates the osteogenic differentiation of MC3T3-E1 through the PI3K-Akt/miR-34c loop. Sci Rep. 2016;6(1):25578. doi:10.1038/srep25578

11. Cong F, Wu N, Tian X, et al. MicroRNA-34c promotes osteoclast differentiation through targeting LGR4. Gene. 2017;610:1-8. doi:10.1016/j.gene.2017.01.028

12. Liu J, Li D, Sun X, Wang Y, Xiao Q, Chen A. Icariine restores LPS-induced bone loss by downregulating miR-34c level. Inflammation. 2016;39(5):1764-1770. doi:10.1007/s10753-016-0411-6

13. Li X, Tang Y, Jia Z, Zhao X, Chen M. Decreased expression of miR-24 in peripheral plasma of type 2 diabetes mellitus patients associated with diabetic foot ulcer. Wound Repair Regen. 2020;28 (6):728-738. doi:10.1111/wrr.12850

14. Mu S, Hua Q, Jia Y, et al. Effect of negative-pressure wound therapy on the circulating number of peripheral endothelial progenitor cells in diabetic patients with mild to moderate degrees of ischaemic foot ulcer. Vascular. 2019;27(4):381-389. doi:10.1177/17085381 19836360

15. Jones PH, Deng B, Winkler J, et al. Over-expression of miR-34c leads to early-life visceral fat accumulation and insulin resistance. Sci Rep. 2019;9(1):13844. doi:10.1038/s41598-019-50191-3

16. Kong L, Zhu J, Han W, et al. Significance of serum microRNAs in pre-diabetes and newly diagnosed type 2 diabetes: a clinical study. Acta Diabetol. 2011;48(1):61-69. doi:10.1007/s00592-0100226-0

17. Wang SS, Li YQ, Liang YZ, et al. Expression of miR-18a and miR-34c in circulating monocytes associated with vulnerability to type 2 diabetes mellitus and insulin resistance. $J$ Cell Mol Med. 2017;21(12):3372-3380. doi:10.1111/jcmm. 13240

18. Kapusta P, Konieczny PS, Hohendorff J, et al. Negative pressure wound therapy affects circulating plasma microRNAs in patients with diabetic foot ulceration. Diabetes Res Clin Pract. 2020;165:108251. doi:10.1016/j.diabres.2020.108251

19. Lv J, Zhang Z, Pan L, Zhang Y. MicroRNA-34/449 family and viral infections. Virus Res. 2019;260:1-6. doi:10.1016/j.virusres.20 18.11.001

20. Zhou X, Chen H, Zhu L, et al. Helicobacter pylori infection related long noncoding RNA (lncRNA) AF147447 inhibits gastric cancer proliferation and invasion by targeting MUC2 and up-regulating miR-34c. Oncotarget. 2016;7(50):82770-82782. doi:10.18632/ oncotarget.13165

21. Noor S, Zubair M, Ahmad J. Diabetic foot ulcer-A review on pathophysiology, classification and microbial etiology. Diabetes Metab Syndr. 2015;9(3):192-199. doi:10.1016/j.dsx.2015.04.007

22. Ladurner R, Küper M, Königsrainer I, et al. Predictive value of routine transcutaneous tissue oxygen tension (tcpO2) measurement for the risk of non-healing and amputation in diabetic foot ulcer patients with non-palpable pedal pulses. Med Sci Monit. 2010;16 (6):CR273-CR277.

23. Tecilazich F, Dinh T, Pradhan-Nabzdyk L, et al. Role of endothelial progenitor cells and inflammatory cytokines in healing of diabetic foot ulcers. PLoS One. 2013;8(12):e83314. doi:10.1371/journal. pone. 0083314

24. Fu XL, Ding H, Miao WW, Chen HL. Association between cigarette smoking and diabetic foot healing: a systematic review and meta-analysis. Int $J$ Low Extrem Wounds. 2018;17(4):247-257. doi: $10.1177 / 1534734618809583$ 
25. Yang H. Progress in treatment of refractory wound and its challenges we are facing. Zhonghua Shao Shang Za Zhi. 2016;32 (4):193-195. doi:10.3760/cma.j.issn.1009-2587.2016.04.001

26. Giurato L, Meloni M, Izzo V, Uccioli L. Osteomyelitis in diabetic foot: a comprehensive overview. World $J$ Diabetes. 2017;8 (4):135-142. doi:10.4239/wjd.v8.i4.135

27. Aragón-Sánchez J, Lipsky BA, Lázaro-Martínez JL. Gram-negative diabetic foot osteomyelitis: risk factors and clinical presentation. Int $J$ Low Extrem Wounds. 2013;12(1):63-68. doi:10.1177/ 1534734613477423

28. Wang N, Yang BH, Wang G, et al. A meta-analysis of the relationship between foot local characteristics and major lower extremity amputation in diabetic foot patients. $J$ Cell Biochem. 2019;120 (6):9091-9096. doi:10.1002/jcb.28183

29. Chen Y, Ding H, Wu H, Chen HL. The relationship between osteomyelitis complication and drug-resistant infection risk in diabetic foot ulcer: a meta-analysis. Int $J$ Low Extrem Wounds. 2017;16 (3):183-190. doi:10.1177/1534734617728642

30. Cui Y, Lu S, Tan H, Li J, Zhu M, Xu Y. Silencing of long non-coding RNA NONHSAT009968 ameliorates the staphylococcal protein A-inhibited osteogenic differentiation in human bone mesenchymal stem cells. Cell Physiol Biochem. 2016;39(4):1347-1359. doi:10.1159/000447839
31. Tamura M, Uyama M, Sugiyama Y, Sato M. Canonical Wnt signaling activates miR-34 expression during osteoblastic differentiation. Mol Med Rep. 2013;8(6):1807-1811. doi:10.3892/mmr.2013.1713

32. Yang X, Yang J, Lei P, Wen T. LncRNA MALAT1 shuttled by bone marrow-derived mesenchymal stem cells-secreted exosomes alleviates osteoporosis through mediating microRNA-34c/SATB2 axis. Aging. 2019;11(20):8777-8791. doi:10.18632/aging.102264

33. Bae Y, Yang T, Zeng HC, et al. miRNA-34c regulates notch signaling during bone development. Hum Mol Genet. 2012;21(13):2991-3000. doi: $10.1093 / \mathrm{hmg} / \mathrm{dds} 129$

34. Swarbrick S, Wragg N, Ghosh S, Stolzing A. Systematic review of miRNA as biomarkers in Alzheimer's disease. Mol Neurobiol. 2019;56(9):6156-6167. doi:10.1007/s12035-019-1500-y

35. Zhao J, Zhang LX, Wang YT, Li Y, Chen MD. Genetic polymorphisms and the risk of diabetic foot: a systematic review and meta-analyses. Int J Low Extrem Wounds. 2020:1534734620977599. doi:10.1177/1534734620977599

Diabetes, Metabolic Syndrome and Obesity: Targets and Therapy

Dovepress

\section{Publish your work in this journal}

Diabetes, Metabolic Syndrome and Obesity: Targets and Therapy is an international, peer-reviewed open-access journal committed to the rapid publication of the latest laboratory and clinical findings in the fields of diabetes, metabolic syndrome and obesity research. Original research, review, case reports, hypothesis formation, expert opinion and commentaries are all considered for publication. The manuscript management system is completely online and includes a very quick and fair peer-review system, which is all easy to use. Visit http://www.dovepress.com/testimonials.php to read real quotes from published authors.

Submit your manuscript here: https://www.dovepress.com/diabetes-metabolic-syndrome-and-obesity-targets-and-therapy-journal 